\title{
Surface displacements, deformations and gravity changes due to underground heat source
}

\author{
Ladislav BRIMICH, Igor KOHÚT \\ Earth Science Institute, Slovak Academy of Sciences, \\ Dúbravská cesta 9, P. O. Box 106, 84005 Bratislava, Slovak Republic; \\ e-mail: Ladislav.Brimich@savba.sk
}

\begin{abstract}
Thermo-elastic strains and stresses play a considerable role in the stress state of the lithosphere and its dynamics, especially at pronounced positive geothermal anomalies. Topography has a significant effect on ground deformation. Two methods for including the topographic effects in the thermo-viscoelastic model are described. First we use an approximate methodology which assumes that the main effect of topography is due to the distance from the source to the free surface and permits to have an analytical solution very attractive for solving the inverse problem. A numerical solution (for $2 \mathrm{D}$ plain strain case) is also computed using finite element method (FEM). The numerical method allows to include the local shape of the topography in the modeling. In the numerical model the buried magmatic body is represented by a finite volume thermal source. The temperature distribution is computed by the higher-degree FEM. For analytical as well as numerical model solution only the forces of thermal origin are considered. The results show that for the volcanic areas with prominent topography, its effect on the perturbation of the thermo-viscoelastic solution (deformation and total gravity anomaly) can be quite significant. In consequence, neglecting the topography could give erroneous results in the estimated source parameters.
\end{abstract}

Key words: displacements, deformations, gravity changes

\section{Introduction}

A magma intrusion in the Earth's crust will cause effects (for example deformation and gravity changes) related to its mass as well to the pressurization of the chamber due to overfilling or temperature changes. The theory of the thermoelastic phenomena shows that thermo-elastic stresses and deformations can arise in an elastic continuum if an inhomogeneous temperature 
field exists in the media (see e.g., Nowacki, 1962). Thus we can expect various thermoelastic phenomena to occur in some regions like volcanic areas, with an anomalous behaviour of the heat flow. In the geodynamic theory, it is well-known that thermo-elastic strains and stresses play a considerable role in the stress state of the lithosphere and its dynamics, especially in localities with pronounced geothermal anomalies (Combs and Hadley, 1977; Teisseyre, 1986). Therefore, Hvoždara and Rosa (1979, 1980) carried out a theoretical analysis of thermo-elastic deformations of a homogeneous halfspace due to a point or linear source of heat, located at a particular depth in the half-space. They proved that thermo-elastic stresses are of expansive type and that they considerably disturb the normal lithostatic stress, specially near the surface of the half-space. Hvoždara and Brimich (1991) presented basic formulae and the results of numerical calculations for the simplified mathematical models of two important effects due to magmatic bodies in the Earth's lithosphere: a) static thermoelastic deformations, b) static elastic deformations due to upward pressure. The magmatic body is approximated by a finite volume source of heat in the first model and by a concentrated vertical force in the second one. The formulae for gravity anomaly due to non-uniform extension connected with thermo-elastic deformations were derived in Hvoždara and Brimich (1995).

Many of the models with inelastic properties considered so far are analytical. They assume point source of deformation and a flat, horizontal free surface. Volcanoes are commonly associated with significant topographic relief. The approximation of Earth's surface as flat and use half-space solutions can lead to erroneous interpretation of the deformation data (see e.g., Cayol and Cornet, 1998; Williams and Wadge, 1998, 2000; Folch et al., 2000). Williams and Wadge, (1998, 2000) and Cayol and Cornet (1998) pointed out that topography has a significant effect on predicted surface deformation by elastic models in regions of significant relief. According to those studies the interpretation of ground-surface displacements with halfspace models can lead to erroneous estimations. Cayol and Cornet (1998) found that the steeper the volcano, the flatter the vertical displacement field. Folch et al. (2000) demonstrated that this result is dramatically emphasised in the viscoelastic case, where the topography changes substantially both the magnitude and the pattern of the displacement field. They also showed that neglecting the topographic effects may, in some cases, introduce an 
error greater than the one implicit in the point source hypothesis. Those reasons led us to study the effect of topography on surface displacements and gravity changes.

\section{Analytical solution}

The thermo-visco-elastic model presented in Hvożdara (1992) was applied to estimate the volcanic ground deformations. Fundamental equations for the uncoupled thermo-visco-elastic problem for a point heat source located at depth $\zeta$ are given in Nowacki (1962). Thermo-visco-elastic gravity anomaly on the surface is given by Brimich (2000).

Charco et al. (2002) propose a simple method for including topographic effects in a 3D thermo-visco-elastic model that allows the source depth to vary with the relief. On the Fig. 1 is the sketch of the model used in the analytical approach. The Figs 2, 3 and 4 show horizontal, vertical thermoviscoelastic displacement (in $\mathrm{m}$ ) as well as the gravity changes computed for different relaxation times and the static value (thermoelastic case) considering (a) a flat surface, and (b)-(d) axially-symmetric volcanic cone with an average slope of the flanks of $15^{\circ}, 20^{\circ}$ and $30^{\circ}$ respectively. The influence of the topography is visible as the horizontal shift of maximal values of the both displacement components and particularly the gravity changes.

The simple method for evaluating the topographic effect in three-dimensional deformation model is based on the assumption, that the main effect

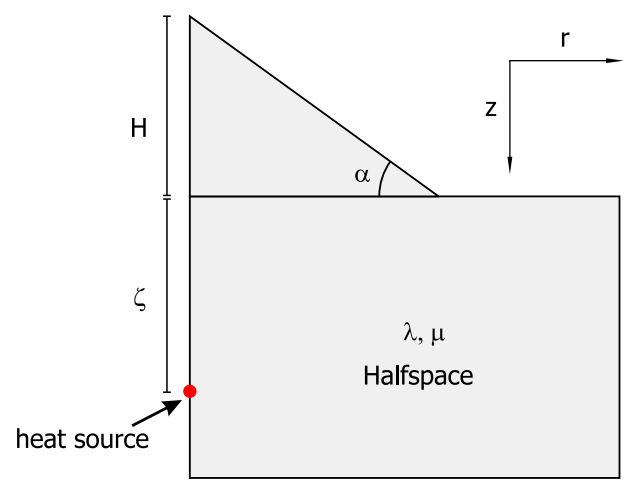

Fig. 1. Sketch of the model used in the analytical approach. 


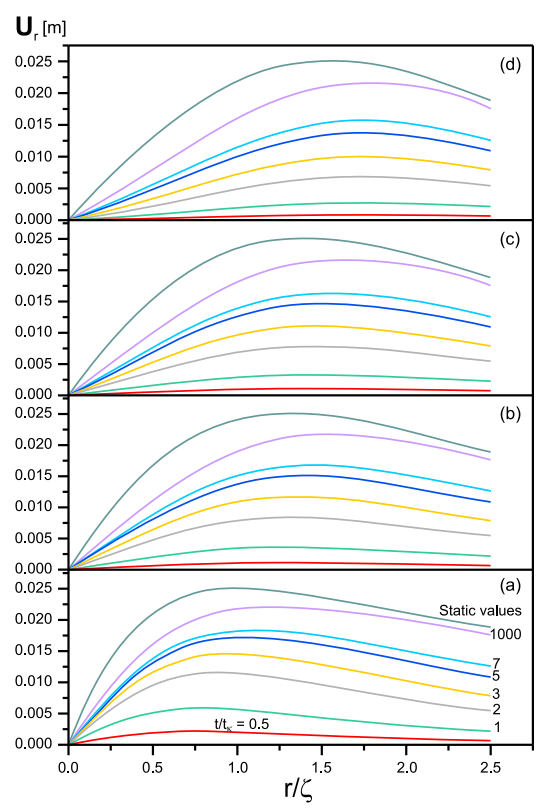

Fig. 2. Radial displacements.

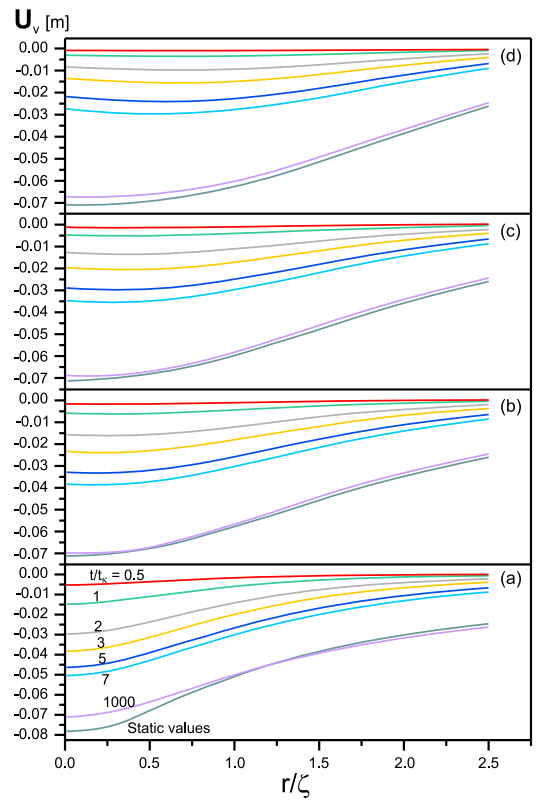

Fig. 3. Vertical displacements.

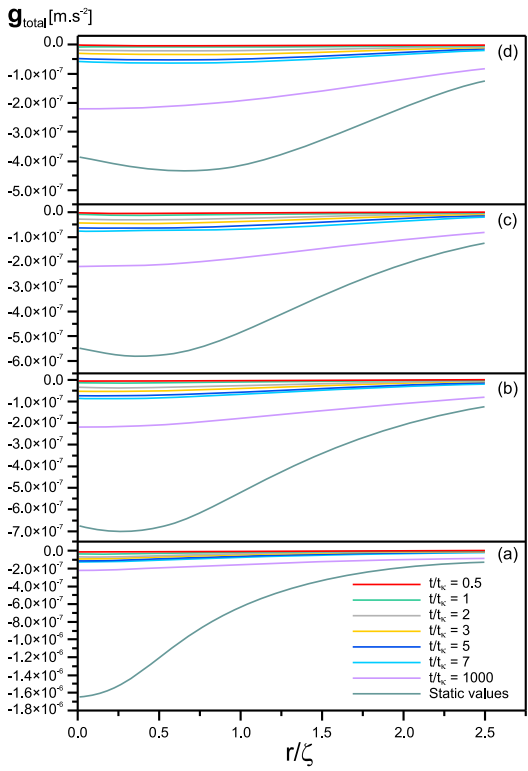

Fig. 4. Gravity changes. 
of the topography is due to the distance from the heat source to the free surface. The solution on the surface is achieved by the consecutive computation of the source influence with corresponding depth of the heat source for the each point. The varying depth methodology was introduced by Williams and Wadge (2000).

\section{Numerical solution}

To include the topography effect in the thermoelastic solution we have used the finite element method computation. The principles and basics of finiteelement method are generally known and are described in numerous monographs (e.g. Irons and Ahmad, 1986; Babuška and Szabo, 1990). All the computations were performed by the COMSOL Multiphysics@ software. Although numerical methods are time consuming, they can handle models with realistic geometry.

The models are homogeneous, isotropic, axially-symmetric with respect to vertical axis. The vertical axis of symmetry is passing through the heat source. Two model versions have been computed - with and without topographical feature modeling the volcano cone (of $2 \mathrm{~km}$ height). The summit of the volcano is located over the thermal source. The domain's horizontal length is $120 \mathrm{~km}$ and the vertical span is from +2 to $-38 \mathrm{~km}$ in order to minimize the influence of the external boundaries. The heat source is modeled by the spherical body in the depth of $5 \mathrm{~km}$. In the computation only the forces of thermal origin are considered.

In the first step, the domain was divided into finite elements. The mesh corresponding to each plane section is formed by 19568 triangular elements. In the neighborhood of the thermal source, the mesh is refined into smaller elements due to the large gradients of computed fields in this area (see Fig. 5). On the Figs. 6 and 7 the radial and vertical displacements obtained by FEM are given.

The finite element method can model more complex models with sources of different geometries and elastic properties of the medium varying with depth. While the analytical approximate methodology can be very attractive for solving the inverse problem, the numerical method described above may be used to include the topography in a more realistic model that permits the consideration of non-uniform elastic and thermal properties of the 


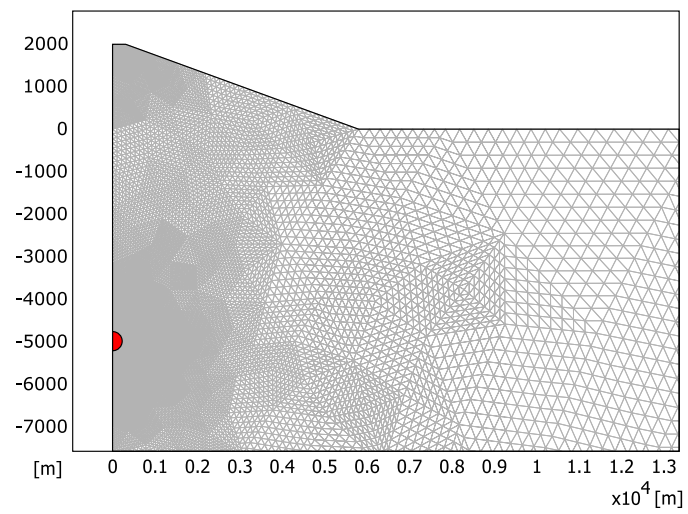

Fig. 5. Mesh with the position of the heat source.

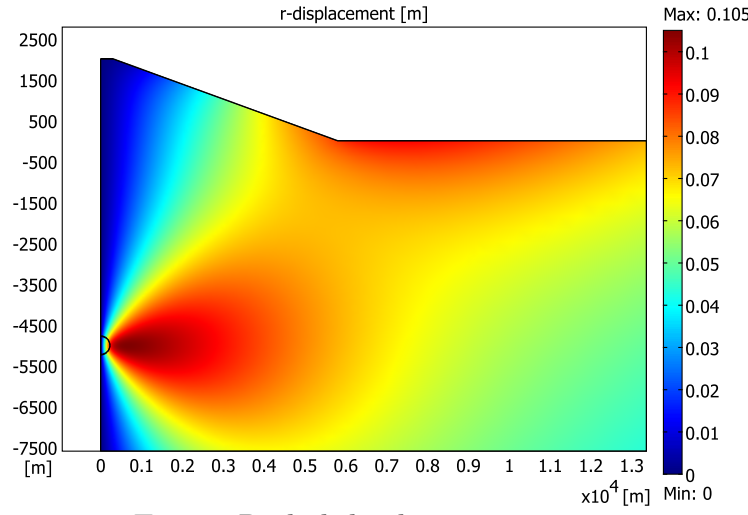

Fig. 6. Radial displacements.

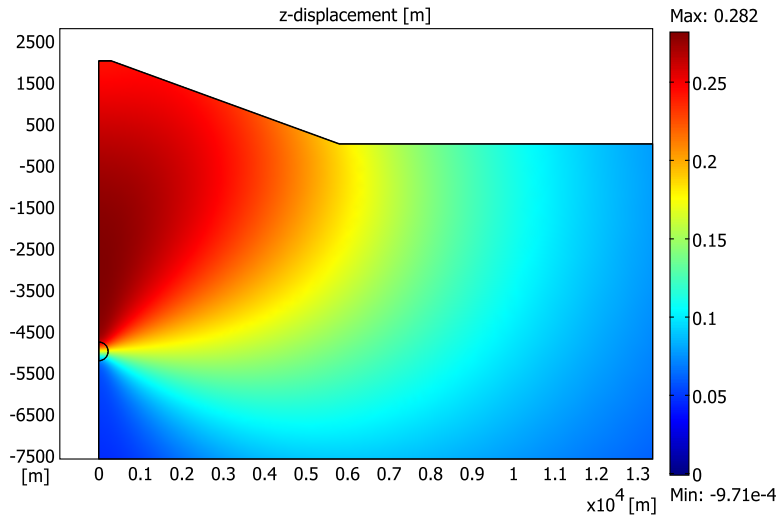

Fig. 7. Vertical displacements. 
medium and the true shape of the Earth's surface. The comparison of the horizontal and vertical displacements for the different models in the Figs. 8 and 9 are given.

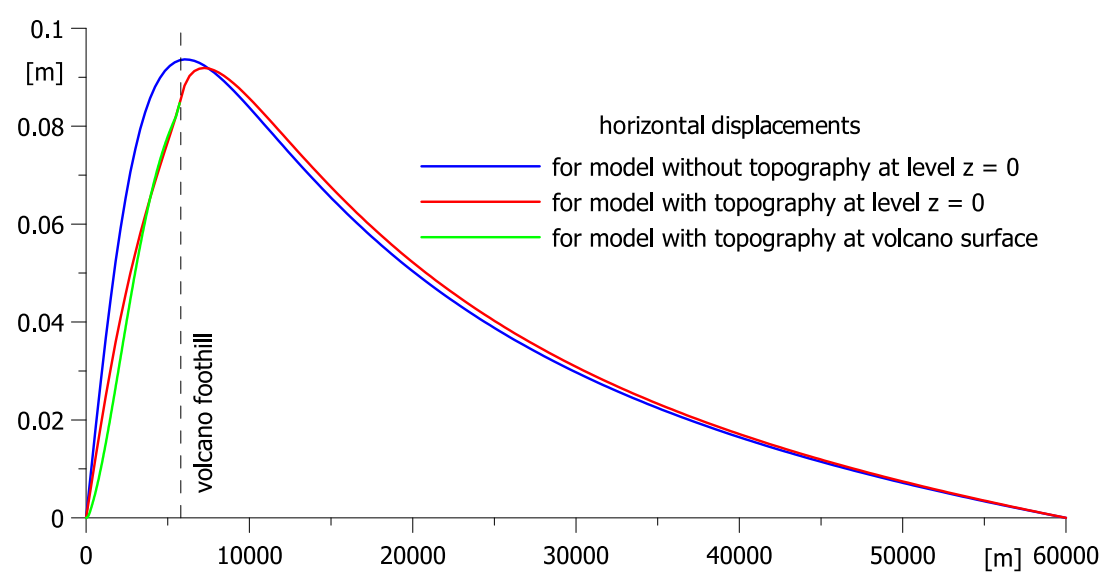

Fig. 8. The comparison of the horizontal displacements for the both variants of the model for the level $z=0(\mathrm{~m})$. The 'foothill' marks the horizontal distance where the volcano topography starts.

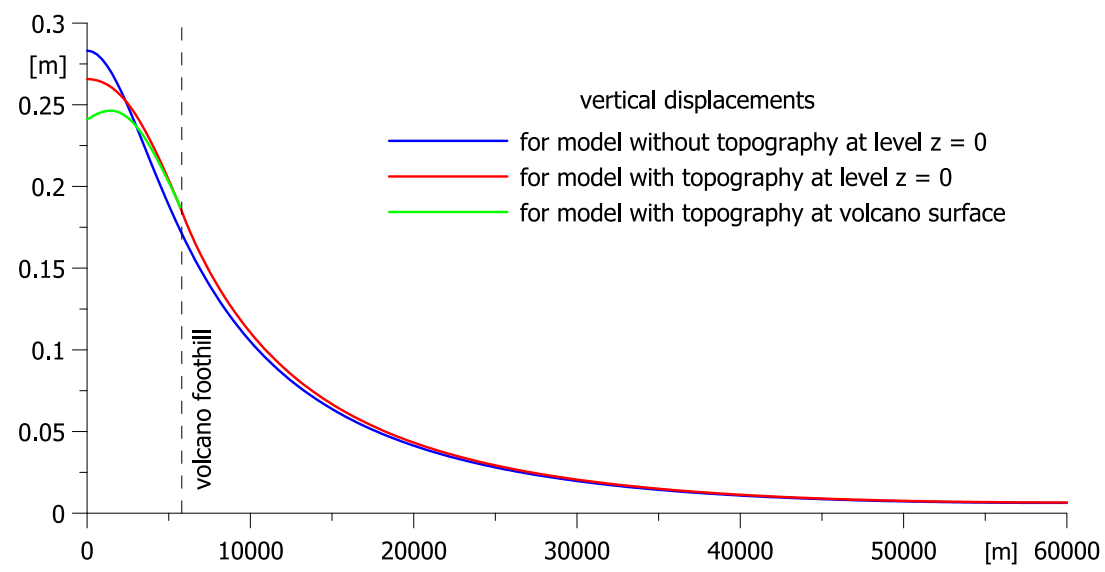

Fig. 9. The comparison of the vertical displacements for the both variants of the model for the level $z=0(\mathrm{~m})$. The 'foothill' marks the horizontal distance where the volcano topography starts. 


\section{Conclusions}

The results show that for the volcanic areas with a pronounced topography the perturbation of the thermo-viscoelastic solution (deformation and total gravity anomaly) due to the topography can be quite significant. In consequence, neglecting topography could give erroneous results in the estimated source parameters. The methods described in this work can be very suitable to more complex models that consider sources of different geometries and allow elastic properties of the medium to vary with depth. While the analytical approximate methodology can be very attractive for solving the inverse problem, the numerical method described above can model the realistic topography and non-uniform elastic and thermal properties of the medium.

Acknowledgements. The authors were supported by Vega Grant Agency under project No. 2/0042/15 and by the Slovak Research and Development Agency (contract No. APVV-16-0482).

\section{References}

Babuška I., Szabo B., 1990: Finite Element Analysis, N. Y., J. Wiley.

Brimich L., 2000: Thermoviscoelastic models of the deformations and gravity changes due to anomalous source of heat. Acta geod. et geoph. Hung., 35, 37-48.

Cayol V., Cornet F. H., 1998: Effects of topography on the interpretation of the deformation field of prominents volcanoes: Application to Etna. Geophys. Res. Lett., 25, 1979-1982.

Charco M., Brimich L., Fernández J., 2002: Topography effects on displacements and gravity changes due to magma intrusions. Geologica Carpathica, 53, 4, 215-221.

Combs J., Hadley D., 1977: Microearthquake investigations of the Mesa feothermal anomaly. Imperial Valley, Califonia. Geophysics, 42, 17-15.

Folch A., Fernández J., Rundle J. B., Marti J., 2000: Ground deformation in a viscoelastic medium composed of a layer overlying a half-space: a comparison between point and extended sources. Geophys. J. Int., 140, 37-50.

Hvoždara M., 1992: Thermo-viscoelastic deformation field due to a point source of heat in the halfspace. Contr. Geophys. Inst. Slov. Acad. Sci., 22, 48-66.

Hvoždara M., Brimich L., 1991: Thermoelastic deformation field due to magmatic bodies. Contr. Geophys. Inst. Slov. Acad. Sci., 21, 59-79. 
Hvoždara M., Brimich L., 1995: Theoretical models for gravity anomalies caused by thermoelastic deformations in the vecinity magmatic bodies. Cahiers du Centre Européen de Géodynamique et Séismologie, 8, 337-349.

Hvoždara M., Rosa K., 1979: Geodynamic effects of thermo-elastic stresses due to a linear heat source. In: V. Babuska and J. Plancar (Eds.): Geodynamic Investigation in Czechoslovakia, Bratislava, Veda, 53-63.

Hvoždara M., Rosa K., 1980: Stresses and displacements due to a stationary point source of heat in an elastic half-space. Studia Geophys. Geod., 24, 51-59.

Irons B., Ahmad S., 1986: Techniques of Finite Elements, Chichester, Ellis Horwood.

Nowacki W., 1962: Thermoelasticity. Oxford, Pergamon Press.

Teisseyre R., 1986: Thermal stresses, In: R. Teisseyre (Ed.): Continuum theories in Solid Earth Physics, Elsevier, Amsterdam.

Williams C. A., Wadge G., 1998: The effect s of the topography on magma chamber deformation models: Application to Mt. Etna and radar interferometry, Geophys. Res. Lett., 25, 1549-1552.

Williams C. A., Wadge G., 2000: An accurate and efficient method for including the effecst of topography in three-dimensional elastic models of ground deformation with applications to radar interferometry. J. Geophys. Res., 105, B4, 8103-8120. 